\title{
Sustainable Reinforced Concrete Beams: Mechanical Optimisation and 3D-Printed Formwork
}

\author{
Sébastien Maitenaz ${ }^{1,2}$, Romain Mesnil ${ }^{1}$, Paul Onfroy ${ }^{2}$, Nicolas Metge ${ }^{2}$, and \\ Jean-François Caron ${ }^{1}$ \\ 1 Laboratoire Navier, Ecole des Ponts ParisTech, Université Gustave Eiffel, CNRS, \\ Marne-La-Vallée, France \\ sebastien.maitenaz@enpc.fr \\ 2 ISC, Vinci Construction France, Chevilly-Larue, France
}

\begin{abstract}
The embodied energy of a building is not only but essentially determined by the quantity of material in its structure. Current fabrication techniques of optimised reinforced concrete (RC) structural elements have not proved sufficient economic competitiveness to be broadly accepted by the construction industry. This partly explains why the construction industry has not yet been able to reduce the embodied energy of newly-constructed buildings or civil works. However, most of the researches driven in the academic world on digital fabrication with concrete are focused on the construction of non-standard structures. The application of digital fabrication to standard structures could help bridge the gap between the need for lower-carbon structures and the economic interest of players in the construction industry.

This paper questions the compatibility of existing building codes with digital manufacturing techniques and presents a novel method for the fabrication of sustainable optimised reinforced concrete beams, compliant with EN 1992-1-1 design requirements.
\end{abstract}

Keywords: Digital Fabrication - Concrete printing - Reinforced Concrete $\cdot$ Structural Optimisation · Strut and Tie Method.

\section{Introduction}

Reinforced concrete (RC) construction, whether it is for buildings or civil works, is still arguably comparable to crafts. Burdensome and time consuming site work directly impacts the design of RC elements. Structural engineers often tend to simplify their design to ease the fabrication process on the one hand, and to avoid possible dramatic fabrication mistakes on the other hand [7. RC structures built at the beginning of the $\mathrm{XX}^{\text {th }}$ century when concrete was an expensive resource, such as bridges from Maillart [4, however prove the possibility to build lighter sustainable structures. Two pathways with different time frames can be explored if we are to save inefficiently consumed concrete. The long term research line concerns the exploration of mechanical optimisation applied to RC elements 
outside the bounds of the current standards both from a purely theoretical [8/2/5] and applied 3 , point of view. In the shorter term, some standard compliant solutions can be found.

This paper presents a new design and fabrication workflow that takes advantage of digital fabrication to manufacture Eurocode 2 compliant optimised beams. By using 3D printed concrete moulds to exclude the hollow parts of trusslike beams, we are able to address the geometric complexity of the formwork. This process drastically reduces the waste as compared to those that would be produced with a traditional formwork. Section 2 introduces the design methodology based on a strut optimisation approach. Section 3 presents the fabrication of a 3-meter-long beam illustrating the feasibility of the proposed method. Eventually, in Section 4 we discuss the potential material savings at stake as well as the levers to enhance the fabrication process in the perspective of its application in the industry.

\section{Design methodology}

\subsection{Computational workflow}

The whole design and fabrication process is automated under the computational workflow presented in Figure1. Based on design requirements, a preliminary EC2 calculation results in the generation of a family of optimal designs from which one is selected based on material savings and manufacturing considerations. A finite element assessment of this design, not included in this paper, could then be provided before the fabrication phase. This paper focuses on the printing of the formwork which is therefore the only criterion considered to assess the constructability of the design. As discussed in Section 4.2 more criteria would have to be taken into account for industrial applications.

\subsection{Reinforced concrete beam design according to EC 2}

Traditional RC beam design involves three main steps. Firstly, longitudinal reinforcements are calculated based on mid-span section equilibrium considerations at ULS, SLS requirements such as maximum displacement, crack opening, rebar and concrete constraints, as well as constraints on the position of the rebars and their total volume. Subsequently, shear reinforcements are calculated to provide minimum shear resistance at ULS. The calculation is performed with discrete values of shear force taking the minimum design value $V_{E d}$ over a beam portion with a length of $z \cot \theta$ (see Figure 3 for notations). Lastly, it is possible to perform longitudinal rebar curtailment. In practice, this step is often neglected so as to simplify the rebar cage preparation. It is worth precising that although minimal steel sections are structural requirements' consequences, effective reinforcement is highly impacted by the geometric constraints (notably the width of the beam) as well as the fabrication method.

As presented in Figure 2, we propose a new EC2 compliant approach for the shear design of RC beams. This approach is detailed in Section 2.3 


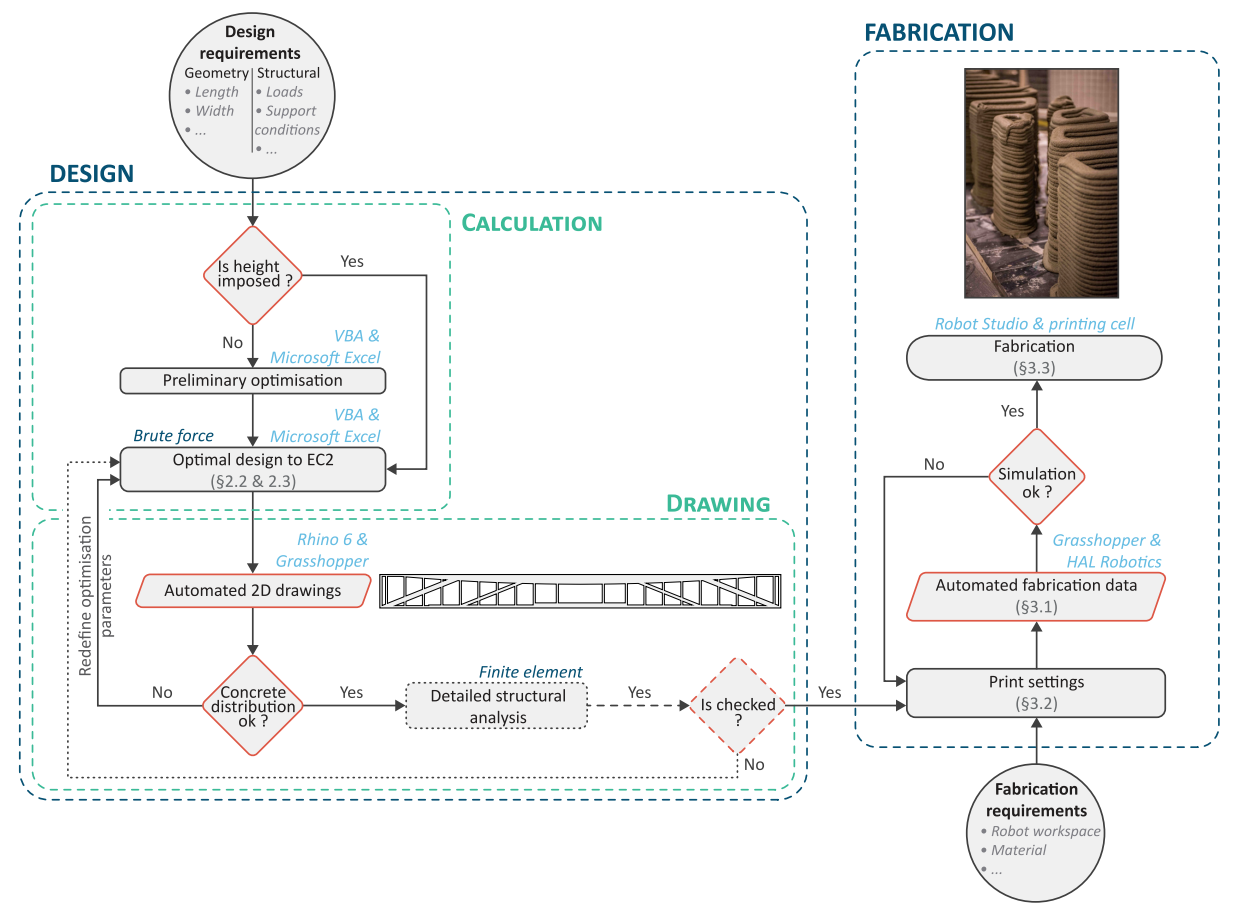

Fig. 1: Computational workflow for the design and fabrication of RC beams according to the strut optimisation approach.

\subsection{Shear strength of reinforced members in the EC 2}

The shear strength design methodology prescribed by the EC2 for RC elements is based on the Variable Strut Inclination Method (VSIM) [1 applied to a strutand-tie model of the given element, see Figure 3. In this method, derived from the first truss analogy models proposed by Ritter [9] and Mörsch [6] the direct contribution of concrete to the shear resistance is ignored. The shear strength of the element is thus given by the minimum between the compressive strength of the concrete (namely the struts, Eq. 1) and the tensile strength of the stirrups (namely the ties, Eq. 2).

$$
\begin{aligned}
V_{R d, \max } & =\sigma_{c} b_{w} \cos \left(\alpha+\theta-\frac{\pi}{2}\right) l_{w} \sin \theta \\
& =\underbrace{a_{c w} \nu_{1} f_{c d}}_{\begin{array}{c}
\text { max. } \\
\text { stress }
\end{array}} \underbrace{b_{w} z \frac{\sin (\alpha+\theta)}{\sin \alpha}}_{\begin{array}{c}
\text { width and height } \\
\text { of the strut }
\end{array}} \sin \theta \\
V_{R d, s} & =\frac{A_{s w}}{s} f_{y w d} z(\cot \theta+\cot \alpha) \sin \alpha
\end{aligned}
$$




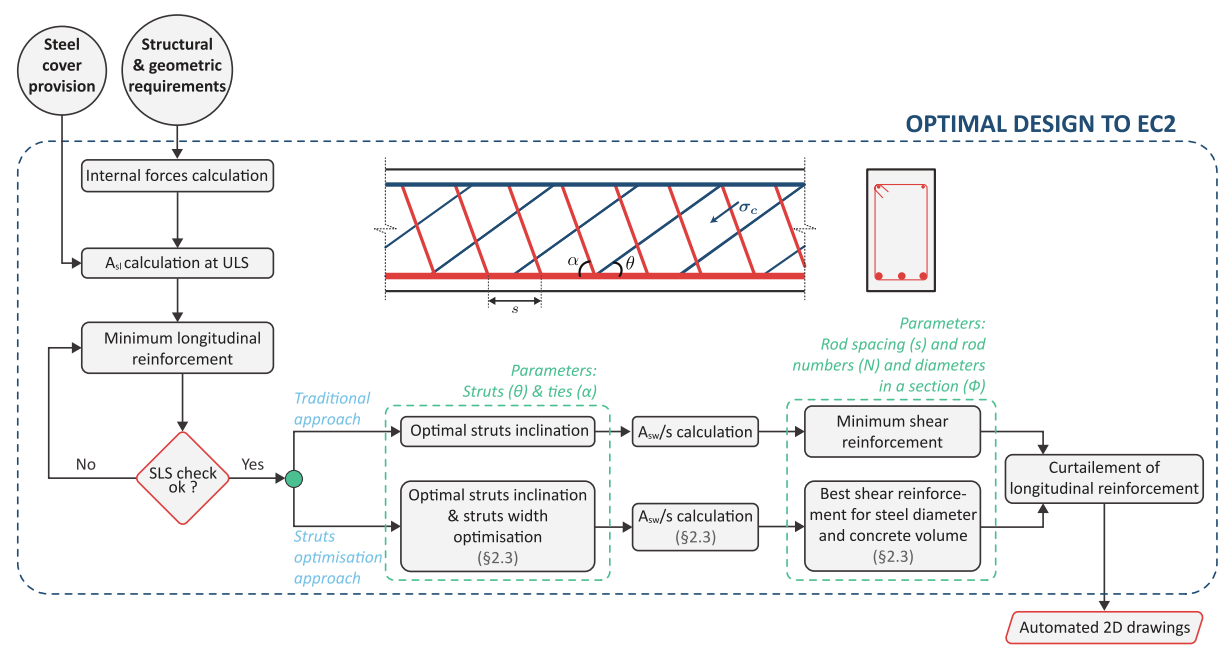

Fig. 2: Eurocode 2 beam design workflow.

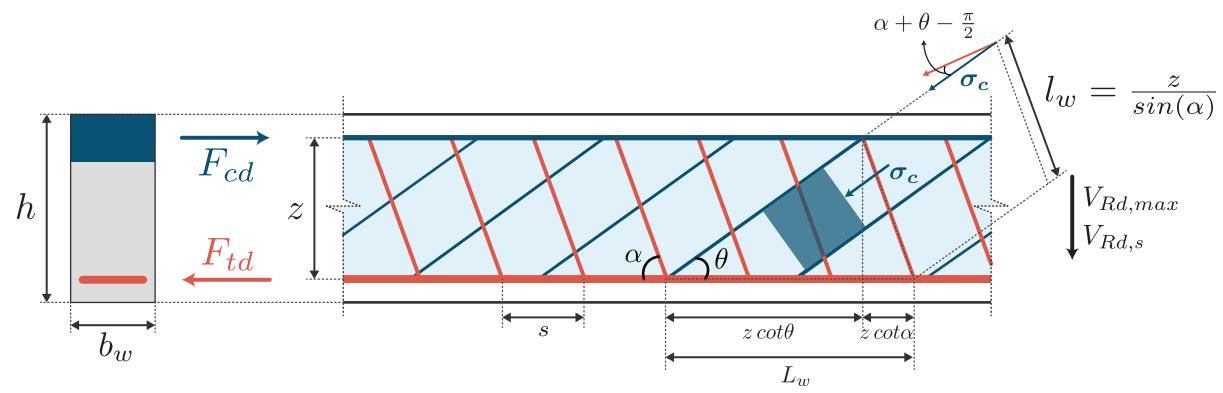

Fig. 3: Eurocode 2 strut-and-tie model for RC elements with shear reinforcements.

In the spirit of the $\mathrm{EC} 2$, an engineer is only given design freedom through the choice of the inclination of both the struts $\left(21,8^{\circ} \leq \theta \leq 45^{\circ}\right)$ and the ties $\left(45^{\circ} \leq \alpha \leq 90^{\circ}\right)$ within some empirically determined limits. All other parameters values are set by the mechanical properties of the materials: $f_{y w d}$ and $f_{c d}$, by the state of stress in the section: $a_{c w}$ and $\nu_{1}$, and by the geometry of the section and the previous design steps: $b_{w}$ and $z$ (inner lever arm for a member with constant depth).

In practice, an engineer would first optimise $A_{s w}$ by properly defining the number and diameter of rods in a section as well as the spacing $s$ between two reinforced section. He would then checks if $V_{E d} \leq V_{R d, \max }$. Otherwise, he would 
increase the strut angle $\theta$. However, as written in Equation 1, the strength of the struts involves the compressive strength of the concrete as well as their width and depth providing one the opportunity to optimise $b_{w}$ and $z$. Our design proposal is based on the struts' height optimisation. The minimum strut height is thus given by Equation 3 . Figure 4 presents a schematic representation of a beam designed with this method and following the steps detailed in Section 2.2.

$$
z_{o p t}=z \cdot \frac{V_{E d}}{V_{R d, \max }}
$$

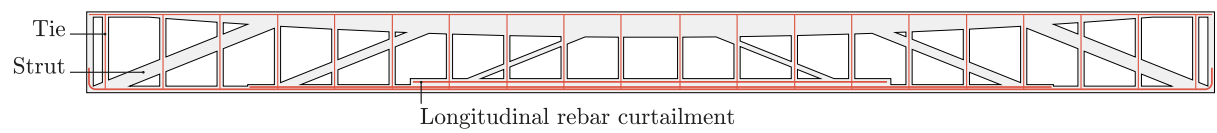

Fig. 4: Schematic representation of a beam designed with the strut optimisation approach.

The next section presents the fabrication of prototype designed with the strut optimisation method.

\section{Case study: Concrete printed formwork for a 3m-long beam}

\subsection{Automated fabrication data generation}

The beams design according to EC2 is carried-out on Excel-based calculation sheets developed by ISC. The results are then exported to Grasshopper. A first set of 'shop' drawings is automatically generated to check the concrete distribution on the one hand and serve as a basis for the fabrication data generation on the other hand. From this stage, everything is managed with the HAL Robotics plug-in using Rhino 6 to visualise the data. Hence, the workflow is fully automated from the calculation to the export of the robot procedure. Figure 5 features some examples of $2 \mathrm{D}$ beam drawings automatically generated with the workflow.

\subsection{Experimental setup and fabrication}

The fabrication process can be divided into two parts: the preparation of the formwork and pouring of the concrete. The formwork contour and lost casing were made with sheets of plywood while the inner formwork pieces where directly printed on the lost casing within our $3 \mathrm{~d}$ concrete printing cell equipped with a Xtree printing head. The Xtree's technology demands to keep a constant time frame between each extruded layer. For such an application, this time constraint raises issues in terms of concrete and accelerator flow rate management as well as 


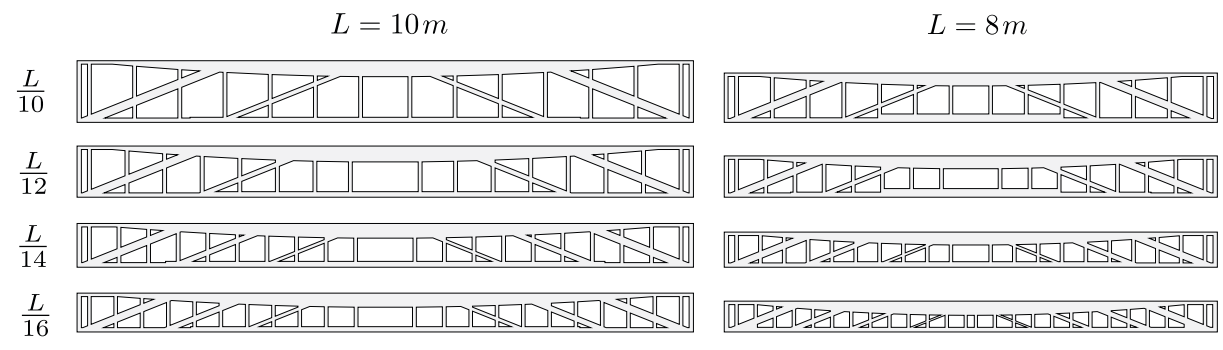

Fig. 5: Benchmark of automatically generated 'shop' drawings.
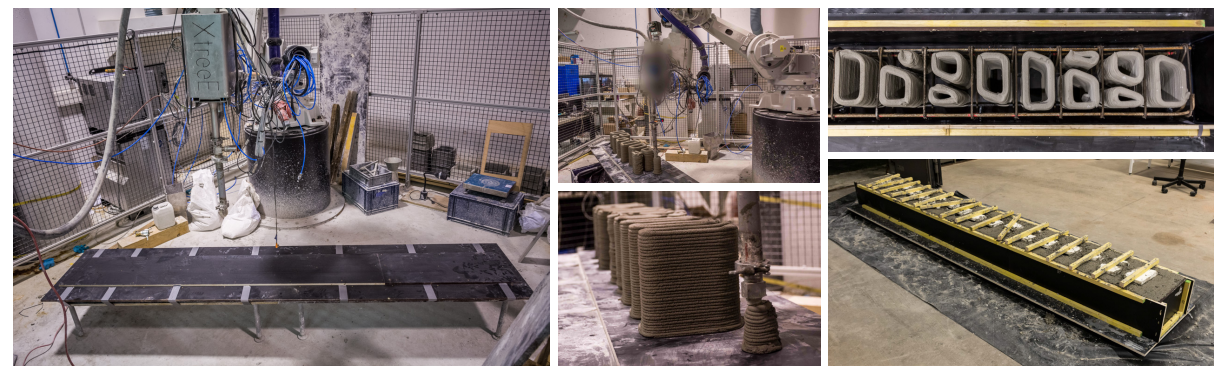

(a) Fabrication of the prototype with, from left to right, preparation of the printing cell, printing of the formwork, final preparation of the formwork and, pouring of the concrete

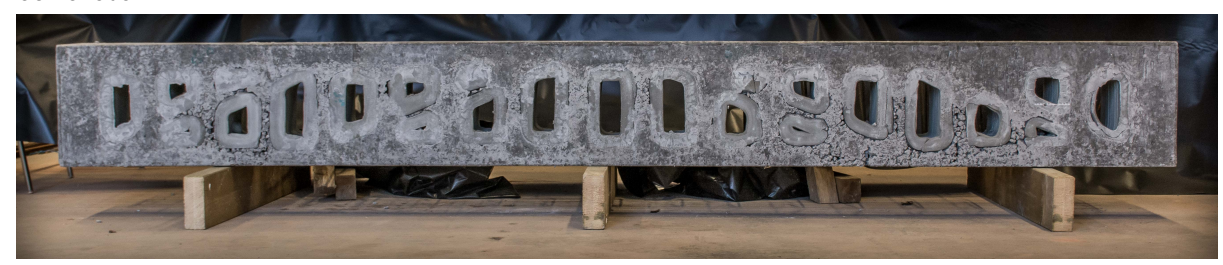

(b) Demoulding of the beam

Fig. 6: Fabrication of the 3m-long beam prototype

for the robot speed. For instance, for the prototype, the ratio between the longest printed piece perimeter and the shortest one was of 4.46. However, pouring concrete remained the biggest challenge. It is well known that the EC2 includes a large amount of rules driven by the need for the concrete to set evenly in the formwork: minimum spacing between the rebars, etc. Here, the narrowness between the rebar cage, the printed pieces and the plywood sheets augments the necessity for carefully formulating the concrete. For this case study, typical distances between the surface of two printed pieces were of $6 \mathrm{~cm}$ with a rebar (HA 6) in between. Our objective was to reach a slump value high enough for allowing us to manually vibrate the concrete and avoid the drift of the blockouts on the lost casing, but low enough to avoid segregation. Table 1 presents the concrete mix, a bill of materials as well as the production rates. 
Table 1: Concrete mix (top left), bill of materials (top right) and production rates (bottom)

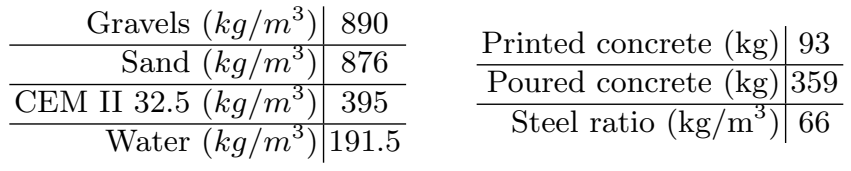

$$
\begin{aligned}
& \begin{array}{r|c}
\text { Printing (min) } & 80 \\
\hline \text { Casting and finishing (min - est.) } & 120 \\
\hline \text { Rebar cage preparation (min - est.) } & 30
\end{array}
\end{aligned}
$$

Despite our attention, the initial concrete mix was too dry: we probably didn't measure the moisture content in the sand and gravels on representative batches. However, we choose not to add too much water in order to guaranty a minimum strength. Finally, we were able to decently pour the mix inside the formwork.

The next section tackles the potential material savings and the fabrication implications at stake.

\section{Discussion on the Potential Material Savings and on the Fabrication Process}

\subsection{Potential material savings with the strut optimisation approach}

Loads and material properties All the beam designs presented in this section were done with the same hypothesis. The beams are considered to be simply supported and are subjected to uniformly distributed dead and live loads. The dead loads include the self-weight of the beam and that of a $17 \mathrm{~cm}$ thick concrete slab with a span of $5 \mathrm{~m}$. The live loads have a characteristic value of $1.5 \mathrm{kN} / \mathrm{m}^{2}$. Appropriate ULS and SLS combinations are taken into account. We consider a concrete of class C30/37 with a density of $2500 \mathrm{~kg} / \mathrm{m}^{3}$ and a steel of class B500B.

Optimisation results Figure 7 presents ratios between concrete volumes in beams designed with the strut optimisation approach and those of their equivalent prismatic beams. The evolution of this ratio with the span is read horizontally while its evolution with the slenderness is read vertically. The smaller the dots and the lighter the shade, the lower the ratio. And, the lower the ratio, the higher the concrete savings.

As expected, the potential concrete savings increase with the span and the slenderness. Some a priori unpredictable peaks can however be observed $(11 \mathrm{~m}$ - L/10 and $12 \mathrm{~m}-\mathrm{L} / 15$ for instance). Those peaks reflect the way the effective shear reinforcements are calculated. As presented in Figure 2, there are three 


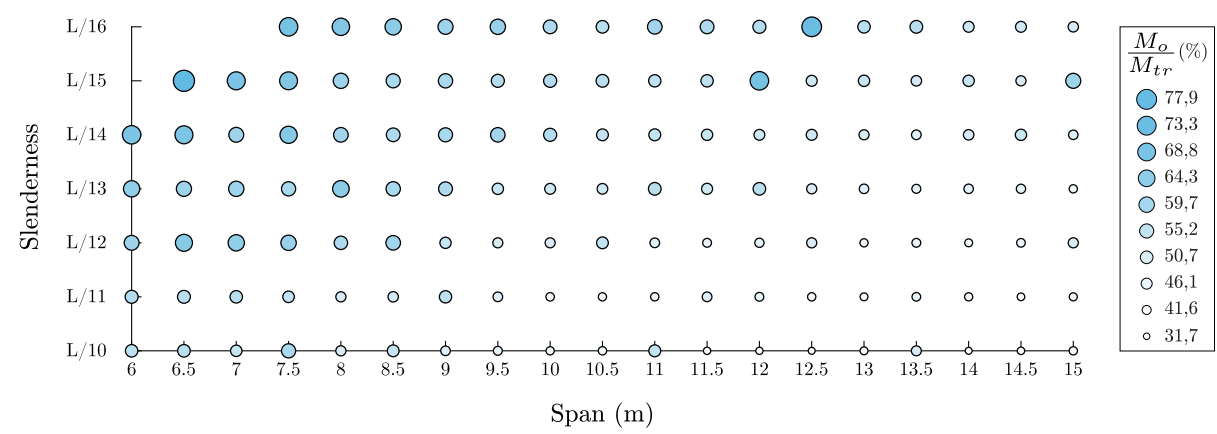

Fig. 7: Evolution of the weight gain with the span and the slenderness $\left(M_{o}\right.$ mass of the optimised beam, $M_{t r}$ - mass of the traditional prismatic beam).

optimisation parameters, the number of rods in a reinforced section, the diameter of those rods and the spacing between two reinforced sections. The peaks are due to particular cases for which the minimum possible effective shear reinforcement was obtained for small spacings between two adjacent reinforced sections. This results in a high consumption of inefficiently used concrete. Figure 8 gives a schematic representation of some beam designs for a span of $12 \mathrm{~m}$. The first beam (L/15) highlights this phenomenon. A reformulation of this problem, in the form of a multi-objective optimisation problem taking into account both the effective steel section and the spacing, allows for a better control of the concrete savings.
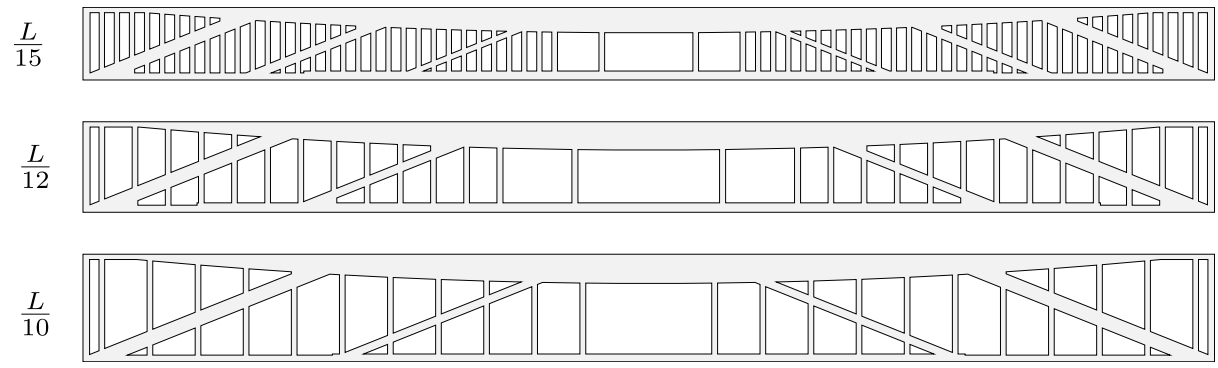

Fig. 8: Examples of beam designs obtained when the only criterion for the optimisation of the ties is the effective steel section.

Concrete savings Most importantly, Figure 7 shows ratios between 31,7 to $77,9 \%$ and thus demonstrates a weight gain in the range of 22 to $68 \%$. In reinforced concrete buildings, beams commonly have a slenderness of about L/10 to $\mathrm{L} / 12$ and a span of 6 to $9 \mathrm{~m}$, a range for which our approach leads to considerable 
savings of about 32 to $58 \%$. Those values of slenderness are not considered to be optimised from a mechanical point of view but they provide an important lever arm. This enables to have low ratios of steel which tends to ease the fabrication process both in terms of assembly of the rebars and pouring of the concrete. But even for lower slenderness, the savings remain important, ranging from 22 to $45 \%$.

These gains need to be seen in perspective. For traditional office buildings, for which the frame is the predominant structural design, there is a direct saving but also an indirect saving through a global reduction of the self weight of the structure. This notably contributes to a reduction of the foundations sizing. For acoustic reasons, traditional housing buildings are more often designed as wallslab structures. From a purely mechanical point of view, this seems nonsensical in the current context. We see here a challenge that remains unsolved.

\subsection{Discussion on the fabrication}

Several technical issues were raised during the construction of the prototype. Firstly, as mentioned in the previous section, the layers' length distribution is critical and would be even more complex to manage for real applications. Typically, for a $7 \mathrm{~m}$-long beam with a slenderness of $\mathrm{L} / 15$, the ratio between the longest printed piece perimeter and the shortest one would be 7.18 . Then, the order of magnitude for the precision of the rebars placement, which were manually assembled on a work site, was ten time higher than that of the formwork (both plywood and concrete parts). It was impossible to insert the rebar cage inside the formwork at first. For future applications, it will be necessary to better control each stage of the process, notably by automating the assembly of the rebars. Similarly, an easily replicable method has yet to be found to prevent the displacement of the printed pieces during the vibration of the formwork without making their retrieval too complicated in the hypothesis of a fully recyclable 3D printed formwork. The use of self-compacting concrete is probably necessary.

From an economical point of view, it is clear that a simpler but more robust printing technology should be used. Typically, a 3-axis robot would be more appropriate. The biggest lever however remains the printed material. Currently, a major part of the formwork cost (both economic and environmental) is in the printed concrete because of its high cement content.

\section{Conclusion \& Perspectives}

Reinforced concrete construction industry is marked by an over and inefficient consumption of concrete. This paper presented a strut optimisation approach, compliant with EC2 requirements at ULS, SLS and for construction detailing. This method results in up to $60 \%$ raw material savings. The associated fabrication process is enabled by digital fabrication and $3 \mathrm{D}$ printing. A $3 \mathrm{~m}$-long prototype was built to prove the constructability of beams designed with the proposed method. 
The construction industry faces major challenges, notably the environmental crisis and the population growth, in the coming years. We believe that it is necessary to find applicable solutions in the very short term. But, the development and transfer of such solutions to the industry will be determined by the ability of all players at stake to rally around a common purpose. Environmental experts are needed to assess the soundness of digital fabrication processes and economists to assess their viability, structural and material specialists are required to propose new ways of building and confirm the proper mechanical behaviour of their proposals, contractors must ensure their implementation at large scale, ... Our proposal is part of this ongoing effort.

Acknowledgment The authors are grateful to Léo Demont, Paul Carneau and Nicolas Ducoulombier for their help during the printing of the formwork. We sincerely thank Christophe Bernard for his technical assistance during the whole fabrication process.

This work was made during Mr. Maitenaz doctorate within the framework of an industrial agreement for training through research (CIFRE number 2018/1055) jointly financed by the company VCF TP IDF SA (Vinci Construction France), and the National Association for Research and Technology (ANRT) of France.

\section{References}

1. EN 1992-1-1: Eurocode 2 - Design of concrete structures - Part 1-1: General rules and rules for buildings. Standard, European committee for standardization, Brussels (2004)

2. Amir, O.: A topology optimization procedure for reinforced concrete structures. Computers \& Structures 114-115, 46-58 (2013). https://doi.org/10.1016/j.compstruc.2012.10.011

3. Asprone, D., Auricchio, F., Menna, C., Mercuri, V.: 3D printing of reinforced concrete elements: Technology and design approach. Construction and Building Materials 165, 218-231 (2018). https://doi.org/10.1016/j.conbuildmat.2018.01.018

4. Billington, D.P.: Robert Maillart and the Art of Reinforced Concrete (1991)

5. Gaynor, A.T., Guest, J.K., Moen, C.D.: Reinforced concrete force visualization and design using bilinear truss-continuum topology optimization 139(4), 607-618 (2013). https://doi.org/10.1061/(ASCE)ST.1943-541X.0000692

6. Mörsch, E.: Der Eisenbetonbau, seine Theorie und Anwendung. Wittwer, Stuttgart (1908)

7. Orr, J., Drewniok, M.P., Walker, I., Ibell, T., Copping, A., Emmitt, S.: Minimising energy in construction: Practitioners' views on material efficiency 140, 125-136 (2019). https://doi.org/10.1016/j.resconrec.2018.09.015

8. Querin, O.M., Victoria, M., Martí, P.: Topology optimization of truss-like continua with different material properties in tension and compression 42(1), 25-32 (2010). https://doi.org/10.1007/s00158-009-0473-2

9. Ritter, W.: Die Bauweise Hennebique (Hennebique's Construction Method). Schweizerische Bauzeitung 33(7), 59-61 (1899) 